\title{
ARE HEALTH WORKERS OF PAEDIATRIC HOSPITALS LESS PRONE TO HEPATITIS B VIRUS INFECTION? A REAPPRAISAL OF A SEROEPIDEMIOLOGICAL SURVEY
}

\author{
Bambino Gesù Children’s Hospital \\ Piazza S. Onofrio, Rome, Italy
Corresponding author:
Vincenzo Di Ciommo
Via F. Baldelli
00146 Rome
Italy
Tel.: + 390668592857
Fax.: + 300668592853 \\ vmaria.diciommo@opbg.net
}

Giuseppe CATANIA, Vincenzo DI CIOMMO

Received: December 5, 2013

Accepted: January 20, 2014

Copyright (C) 2014 by University Clinical Hospital Tuzla. E-mail for permission to publish: paediatricstoday@ukctuzla.ba

\begin{abstract}
Objective - Health care workers (HCWs) are considered at risk of hepatitis $B$ virus (HBV) infection, but the risk in those who work in paediatric hospitals has received little attention. The primary objective was to assess the prevalence of $\mathrm{HBV}$ infection among paediatric HCWs in comparison to those of general hospitals, on the assumption that the former are less exposed due to a lower prevalence in paediatric patients. Methods - Data from a pre-vaccination era were retrieved, and a cross-sectional study conducted in the setting of the vaccination campaign conducted in Latium Region (Italy) to prevent hepatitis $\mathrm{B}$ in HCWs. HBsAg and anti-HBsAg were tested in 1,894 HCWs of paediatric and general hospitals (1,178 and 716, respectively). Multiple logistic regression was performed to adjust for confounders related to professional and personal variables. Results - Overall prevalence in HCWs of HBV infection was $16.8 \%$ (95\% confidence limits, CL: 15.2-18.6). General hospitals carried a significantly higher risk of $\mathrm{HBV}$ infection than paediatric hospitals (OR 1.77, 95\% CL 1.35-2.31) after adjustment for confounding factors employment, department, working years, recent needle injury, and birth cohort, the latter being the only personal risk factor that added significantly to the effect of professional characteristics. Nurses and physicians were more exposed to needle injury, which was an independent significant risk factor of HBV infection (OR 1.60, 95\%CL 1.19-2.14). Conclusions - HCWs of paediatric hospitals are less at risk of HBV infection than general hospitals.
\end{abstract}

Key words: Hepatitis B virus - Health care workers • Pediatric hospital.

HBV can be transmitted from an infected mother to the infant, through sexual intercourse, and by percutaneous exposure to infectious blood or body fluids, including transfusions, injections or other procedures with infected equipment, e.g. needle injuries incurred by Health Care Workers (HCWs) (3). It has been estimated that 65,600 infections from $\mathrm{HBV}$ can be attributed worldwide 
to sharp injuries among HCWs (4). Between $29.3 \%$ of HCWs in central Italy (5) and $32.0 \%$ in a UK district hospital (6) reported at least one needle injury in the preceding year, and in two paediatric hospitals 5.4$6.5 \%$ of HCWs per year were reported in a surveillance setting (7-9). Professional risk is directly associated with prevalence of infections among patients (10), the frequency of the exposure and the probability of transmission. Western Europe is a low prevalence area, particularly for females $(<2 \%)$, but between 1990 and 2005 an increase was observed (11) while in Central and Eastern Europe children remain the most affected by HBV infection with a prevalence between 4 and 5\% (11).

Italy has an intermediate general prevalence of HBsAg (2-7\%) (1): a survey conducted before introduction of vaccination showed an HBsAg prevalence in navy recruits of $3.4 \%$ and of any HBV marker of $17.7 \%$, with signs of multiple injections, southern region of residence and low education as the main risk factors (12). A much lower prevalence of HBsAg - $0.4 \%$ - was found in Italy in pre-vaccination era in the paediatric age group (13).

It was our aim to reappraise the serological survey in a population of HCWs in order to elucidate prevalence of $\mathrm{HBV}$ infection and the risk factor of exposure to a paediatric population, presumed to carry a lower risk, in comparison of an adult one

\section{Subjects and methods}

The data reported here are part of a seroepidemiological survey implemented as a first step of a vaccination campaign, and consequently the design is cross-sectional. The campaign was organized and funded by Regione Lazio, against hepatitis B for at risk subgroups of population: HCWs, newborn of $\mathrm{HBsAg}$-positive mothers, patients on dialysis, sexual partners of HBsAg carriers, and was conducted between 1985 and 1995 (approximately).

The HBV screening of $\mathrm{HCW}$ was at the time of the investigation mandatory. A questionnaire was filled for each HCW comprising demographic and employment data, past history of hepatitis, transfusions, major surgery, dental cures (in the last 6 months), needle-stick injuries, aspecific or specific anti-HBV immunoglobulin. A blood sample was tested for HBsAg and anti-HbsAg (ELI$S A$ radioimmunoassay, Abbott) and alanineaminotransferase (ALT).

Paediatric hospitals were presumed to be at low-risk for HBV transmission and their HCWs were considered as unexposed, whereas HCWs of general hospitals were considered exposed. With a ratio of unexposed/exposed 1.6:1 (1,178 versus 716) we can detect with an alpha of 5\% (significance level of 0.05 ) a difference between a prevalence of $20 \%$ in exposed and $10 \%$ in unexposed with a power of about $80 \%$ (1-beta, that represents a $20 \%$ probability to miss a difference as statistically significant).

\section{Setting}

The Bambino Gesù Children's Hospital, a 611-beds teaching institution is a referral hospital for child patients from middle and southern Italy. It has been the referral centre for vaccination of newborns and one of the vaccination centres for screening and vaccination of HCWs; it has provided screening and vaccination for its personnel, for another small paediatric hospital with 40 beds, and for two acute-care general hospitals (with 370 and with 323 beds, respectively). All the four hospitals are in Rome.

\section{Screening procedures}

Inclusion criteria: all HCWs, comprising: all nurses, all personnel of surgical theatres, all personnel of laboratory, pathology and $\mathrm{x}$ - 
rays departments, blood banks, and all physicians. University graduates with clinical involvement were grouped separately as "Other clinicians". Midwives were included in the category of professional nurses. Dieticians, physiotherapists and other HCWs involved with children's rehabilitation were combined in the category of technicians; the "other" category consisted of social workers, manual skilled workers with access to medical devices, and ambulance drivers. Outpatient clinic includes HCWs devoted to blood sampling for laboratory exams. After completion of the first vaccination campaign the procedure was extended to include student nurses successively enrolled in the Nursing School and to other HCWs who were hired. Student nurses were grouped in the "other" category of hospital department.

Exclusion criteria: any person working in the hospital not as an HCW (e.g. clerk without contact with patients or wards or clinic, personnel of kitchens). HCWs were invited to the vaccination unit for screening. HCWs who tested negative were therefore candidate for vaccination. A "positive" test for antiHBsAg was considered a test with an antiHBsAg titre $\geq 10 \mathrm{IU} / \mathrm{ml}$. Subjects with an equivocal anti-HBsAg titre between 10 and 19 , considered a "positive" test of past infection by $\mathrm{HBV}$ were nevertheless considered as candidates for vaccination, because these were more likely to be "false positives"; in addition presence of antibodies was not regarded a contraindication to vaccination. People negative for $\mathrm{HBsAg}$ or with anti-HBsAg $<20$ $\mathrm{IU} / \mathrm{ml}$, that was considered potentially not protective were considered eligible for vaccination.

\section{Ethics statement}

Data were anonymised and ethical approval was obtained from Ethical Committee of the paediatric hospital.

\section{Statistical analysis}

Statistical software STATA (8.0) was used for all analyses. After univariate description of demographic and professional exposures, logistic multiple regression was performed with HBV infection or immunity (HbsAg or anti-HB positive) as the primary outcome of interest. The main exposure of interest was the type of the hospital (general versus paediatric), adjusting for professional and personal characteristics. For the primary outcome, HBV infection, a second, simpler model was fitted.

\section{Results}

A total of 1,178 HCWs were screened, 62.2\% of whom were from the paediatric hospitals. After the screening 319 infected subjects were excluded from vaccination because of $\mathrm{HBsAg}$ or anti-HBsAg positivity (with a titre considered protective. i.e. $\geq 20$ IU).

Major differences existed between HCWs of general and paediatric hospitals: among the former there were fewer student nurses, less personnel in Neonatology Departments, a longer length of employment. In addition, HCWs in general hospitals were more likely to be single, male, older, more exposed to past surgery, transfusions and recent dental care (Table 1). As most differences can be connected with different risks of HBV infection all were considered potential confounders.

Yearly incidence of at least one needle injury among HCWs was $23.5 \%$, with no significant difference between paediatric and general hospitals (Table 1); HCWs with direct contact with patients' care (nurses and physicians) and surgical environments carried the highest risk (Table 2). Only 24.1\% of the subjects who reported a history of recent needle injury received anonspecific or specific anti-HBV immunoglobulins. 
Table 1 Professional and personal characteristics by type of hospital

\begin{tabular}{|c|c|c|c|c|}
\hline Characteristics & $\begin{array}{l}\text { Paediatric hospital } \\
\mathrm{n}(\%)\end{array}$ & $\begin{array}{l}\text { General hospital } \\
\mathrm{n}(\%)\end{array}$ & $\begin{array}{l}\text { Total } \\
\text { (n) }\end{array}$ & p-value* \\
\hline \multicolumn{5}{|l|}{ Employment } \\
\hline Nurses & 458 (38.9) & $359(50.1)$ & 817 & \multirow{6}{*}{$<0.001$} \\
\hline Student nurse & $262(22.2)$ & $65(9.1)$ & 327 & \\
\hline Porters and other & $177(15.0)$ & $93(12.9)$ & 269 & \\
\hline Technicians & $63(5.4)$ & $55(7.7)$ & 118 & \\
\hline Physicians & $191(16.2)$ & $135(18.9)$ & 326 & \\
\hline Other clinicians & $27(2.3)$ & $10(1.4)$ & 37 & \\
\hline \multicolumn{5}{|l|}{ Service } \\
\hline Surgical & $284(24.1)$ & $264(36.9)$ & 548 & \multirow{6}{*}{$<0.001$} \\
\hline Medical & $197(16.7)$ & $120(16.8)$ & 317 & \\
\hline Neonatology & $232(19.7)$ & $56(7.8)$ & 288 & \\
\hline Laboratory & $79(6.7)$ & $81(11.3)$ & 160 & \\
\hline Other & $355(30.1)$ & $157(21.9)$ & 512 & \\
\hline Outpatient clinic & $31(2.6)$ & $38(5.3)$ & 69 & \\
\hline \multicolumn{5}{|l|}{ Years of work } \\
\hline $1-9$ & $770(65.4)$ & $378(52.8)$ & 1,148 & \multirow{3}{*}{$<0.001$} \\
\hline $10-19$ & $339(28.8)$ & $249(34.8)$ & 588 & \\
\hline$\geq 20$ & $69(5.9)$ & $89(12.4)$ & 158 & \\
\hline \multicolumn{5}{|l|}{ Needle injury } \\
\hline No & $773(65.6)$ & $456(63.7)$ & 1,229 & \multirow{3}{*}{0.68} \\
\hline Yes & $270(22.9)$ & $175(24.4)$ & 445 & \\
\hline Do not remember & $135(11.5)$ & $85(11.9)$ & 220 & \\
\hline \multicolumn{5}{|l|}{ Sex } \\
\hline Females & $976(82.9)$ & $427(59.6)$ & 1,403 & \multirow{2}{*}{$<0.001$} \\
\hline Males & $202(17.2)$ & $289(40.4)$ & 491 & \\
\hline \multicolumn{5}{|l|}{ Education } \\
\hline Low intermediate & $443(37.6)$ & $311(43.4)$ & 754 & \multirow{3}{*}{0.002} \\
\hline Higher intermediate & $517(43.9)$ & $258(36.3)$ & 775 & \\
\hline University & $218(18.5)$ & $147(20.5)$ & 365 & \\
\hline \multicolumn{5}{|l|}{ Marital status } \\
\hline Single & $609(51.7)$ & $259(36.2)$ & 868 & \multirow{3}{*}{$<0.001$} \\
\hline Married & $532(45.2)$ & $434(60.6)$ & 966 & \\
\hline Divorced/widow & $37(3.1)$ & $23(3.2)$ & 60 & \\
\hline \multicolumn{5}{|l|}{ Year of birth } \\
\hline $1960-74$ & $459(39.0)$ & $134(18.7)$ & 593 & \multirow{4}{*}{$<0.001$} \\
\hline $1950-59$ & $398(33.8)$ & $277(38.7)$ & 675 & \\
\hline $1940-49$ & $219(18.6)$ & $193(26.0)$ & 412 & \\
\hline 1916-39 & $102(8.7)$ & $112(15.6)$ & 214 & \\
\hline \multicolumn{5}{|l|}{ Transfusions } \\
\hline No & $1,086(92.2)$ & $674(94.1)$ & 1,760 & \multirow{3}{*}{0.027} \\
\hline Yes & $49(4.2)$ & $31(4.3)$ & 80 & \\
\hline Do not remember & $43(3.7)$ & $11(1.5)$ & 54 & \\
\hline
\end{tabular}




\section{Continuation of Table 1 Professional and personal characteristics by type of hospital}

\begin{tabular}{|c|c|c|c|c|}
\hline Characteristics & $\begin{array}{l}\text { Paediatric hospital } \\
\mathrm{n}(\%)\end{array}$ & $\begin{array}{l}\text { General hospital } \\
\mathrm{n}(\%)\end{array}$ & $\begin{array}{l}\text { Total } \\
\text { (n) }\end{array}$ & p-value* \\
\hline \multicolumn{5}{|l|}{ Surgery } \\
\hline No & $1,010(85.7)$ & $603(84.2)$ & 1613 & \multirow{3}{*}{$<0.001$} \\
\hline Yes & $123(10.4)$ & $108(15.1)$ & 231 & \\
\hline Do not remember & $45(3.8)$ & $5(0.7)$ & 50 & \\
\hline \multicolumn{5}{|l|}{ Teeth care } \\
\hline No & $837(71.1)$ & $578(80.7)$ & 1,415 & \multirow{3}{*}{$<0.001$} \\
\hline Yes & $306(26.0)$ & $132(18.4)$ & 438 & \\
\hline Do not remember & $35(3.0)$ & $6(0.8)$ & 41 & \\
\hline Total & $1,178(100)$ & $716(100)$ & 1,894 & \\
\hline
\end{tabular}

${ }^{*}$ Chi-square test.

Table 2 Needle injuries (last year) by professional characteristics

\begin{tabular}{|c|c|c|c|c|c|}
\hline $\begin{array}{l}\text { Professional } \\
\text { characteristics }\end{array}$ & HCWs* & $\begin{array}{l}\text { Needlestick } \\
\mathrm{n}(\%)\end{array}$ & $\begin{array}{l}\text { No Needlestick } \\
\mathrm{n}(\%)\end{array}$ & $\begin{array}{l}\text { Does not remember } \\
\mathrm{n}(\%)\end{array}$ & p-value** \\
\hline \multicolumn{6}{|l|}{ Employment } \\
\hline Nurses & 817 & $244(29.9)$ & $489(59.9)$ & $84(10.3)$ & \multirow{6}{*}{$<0.001$} \\
\hline Student nurse & 327 & $25(7.7)$ & $248(75.8)$ & $54(16.4)$ & \\
\hline Porters \& other & 269 & $47(17.5)$ & $205(76.2)$ & $17(6.3)$ & \\
\hline Technicians & 118 & $22(18.6)$ & $90(76.3)$ & $6(5.1)$ & \\
\hline Physicians & 326 & 103 (31.6) & $167(51.2)$ & $56(17.2)$ & \\
\hline Other clinicians & 37 & $4(10.8)$ & $30(81.1)$ & $3(8.1)$ & \\
\hline \multicolumn{6}{|l|}{ Service } \\
\hline Surgical & 548 & $183(33.4)$ & $305(55.7)$ & $60(11.0)$ & \multirow{6}{*}{$<0.001$} \\
\hline Medical & 317 & $76(24.0)$ & $208(65.6)$ & $33(10.4)$ & \\
\hline Neonatology & 288 & $70(24.3)$ & $184(63.9)$ & $34(11.8)$ & \\
\hline Laboratory & 160 & $34(21.3)$ & $111(69.4)$ & $15(9.4)$ & \\
\hline Other & 512 & $66(12.9)$ & $375(73.2)$ & 71 (13.9) & \\
\hline Outpt. clinic & 69 & $16(23.2)$ & $46(66.7)$ & $7(10.1)$ & \\
\hline \multicolumn{6}{|l|}{ Years of work } \\
\hline $1-9$ & 1,148 & $249(21.7)$ & $769(67.0)$ & $130(11.3)$ & \multirow{3}{*}{0.14} \\
\hline $10-19$ & 588 & $155(26.4)$ & $365(62.1)$ & 68 (11.6) & \\
\hline$>20$ & 158 & $41(26.0)$ & $95(60.1)$ & 22 (13.9) & \\
\hline Total & 1,894 & $445(23.5)$ & $1,229(64.9)$ & 220 (11.6) & \\
\hline
\end{tabular}

${ }^{*}$ Health care workers; ${ }^{* *}$ Chi-square test.

A history of hepatitis B is a poor predictor of serum prevalence of HBV markers: it was elicited only from $9.0 \%$ of the HCWs, and of specific history of hepatitis B only from
2.5\%, without difference between different hospitals. Abnormal ALT was found in 38 subjects $(2.0 \%)$, none of whom was HBsAgpositive. 


\section{HBV infection}

HCWs in general hospitals showed an approximately twofold odds ratio (OR) of a past $\mathrm{HBV}$ infection compared with HCWs of the paediatric hospitals (Table 3). A clear correlation was observed between longer duration of employment and higher prevalence of HBV infection; a similar relationship is shown with birth cohort.

Table 3 HBV markers in Health care workers by professional characteristics

\begin{tabular}{|c|c|c|c|c|c|c|c|}
\hline $\begin{array}{l}\text { Professional } \\
\text { characteristics }\end{array}$ & HCWs* & $\begin{array}{l}\text { HbsAg+ } \\
\text { n (\%) }\end{array}$ & $\begin{array}{l}\text { Anti-Hbs+ } \\
\text { n (\%) }\end{array}$ & $\begin{array}{l}\text { Any } \\
\text { marker + } \\
\mathrm{n}(\%)\end{array}$ & $\begin{array}{l}\text { OR }(95 \% \text { CL) } \\
\text { any marker + }\end{array}$ & $\begin{array}{l}\text { Adjusted OR } \\
(95 \% \text { CL) of any } \\
\text { marker + Full } \\
\text { model }\end{array}$ & $\begin{array}{l}\text { Adjusted OR ( } 95 \% \\
\text { CL) of any marker } \\
\text { + Simpler model }\end{array}$ \\
\hline \multicolumn{8}{|l|}{ Hospital } \\
\hline Paediatric & 1,178 & $16(1.4)$ & $131(11.1)$ & $147(12.5)$ & 1 & 1 & 1 \\
\hline General & 716 & $20(2.8)$ & $152(21.2)$ & $172(24.0)$ & $2.22(1.73-2.84)$ & $1.73(1.30-2.29)$ & $1.77(1.35-2.31)$ \\
\hline \multicolumn{8}{|l|}{ Employment } \\
\hline Nurses & 817 & $21(2.6)$ & $158(19.3)$ & $179(21.9)$ & 1 & 1 & 1 \\
\hline $\begin{array}{l}\text { Student } \\
\text { nurse }\end{array}$ & 327 & $3(0.9)$ & $17(5.2)$ & $20(6.1)$ & $0.23(0.14-0.38)$ & $0.47(0.26-0.84)$ & $0.48(0.27-0.84)$ \\
\hline $\begin{array}{l}\text { Porters and } \\
\text { other }\end{array}$ & 269 & $8(3.0)$ & $38(14.1)$ & $46(17.1)$ & $0.74(0.51-1.05)$ & $0.52(0.34-0.80)$ & $0.56(0.38-0.85)$ \\
\hline Technicians & 118 & $3(2.5)$ & $18(15.2)$ & $21(17.8)$ & $0.77(0.47-1.27)$ & $0.66(0.35-1.24)$ & $0.67(0.37-1.23)$ \\
\hline Physicians & 326 & $1(0.3)$ & $48(14.7)$ & $49(15.0)$ & $0.63(0.45-0.89)$ & $0.52(0.26-1.02)$ & $0.46(0.32-0.68)$ \\
\hline $\begin{array}{l}\text { Other } \\
\text { clinicians }\end{array}$ & 37 & $-)$ & $4(10.8)$ & $4(10.8)$ & $0.43(0.15-1.24)$ & $0.54(0.15-1.89)$ & $0.41(0.13-1.32)$ \\
\hline \multicolumn{8}{|l|}{ Service } \\
\hline Surgical & 548 & $13(2.4)$ & $76(13.9)$ & $89(16.2)$ & 1 & 1 & 1 \\
\hline Medical & 317 & $5(1.6)$ & $51(16.1)$ & $56(17.7)$ & $1.11(0.77-1.60)$ & $1.29(0.87-1.91)$ & $1.23(0.84-1.82)$ \\
\hline Neonatology & 288 & $6(2.1)$ & $45(15.6)$ & $51(17.7)$ & $1.11(0.76-1.62)$ & $1.37(0.91-2.06)$ & $1.33(0.89-2.00)$ \\
\hline Laboratory & 160 & $2(1.2)$ & $31(19.4)$ & $33(20.6)$ & $1.34(0.86-2.09)$ & $1.63(0.93-2.83)$ & $1.59(0.92-2.76)$ \\
\hline Other & 512 & $8(1.6)$ & $61(11.9)$ & $69(13.5)$ & $0.80(0.57-1.13)$ & $1.64(1.11-2.44)$ & $1.57(1.06-2.32)$ \\
\hline $\begin{array}{l}\text { Outpatient } \\
\text { clinic }\end{array}$ & 69 & $2(2.90)$ & $19(27.5)$ & $21(30.4)$ & $2.26(1.28-3.97)$ & $1.16(0.62-2.15)$ & $1.19(0.65-2.18)$ \\
\hline \multicolumn{8}{|l|}{ Years of work } \\
\hline $1-9$ & 1,148 & $7(0.6)$ & 114 (9.9) & $121(10.5)$ & 1 & 1 & 1 \\
\hline $10-19$ & 588 & $22(3.7)$ & $116(19.7)$ & $138(23.5)$ & $2.60(1.98-3.42)$ & $1.48(1.15-1.91)^{* *}$ & $1.57(1.23-2.00)^{* *}$ \\
\hline$\geq 20$ & 158 & $7(4.4)$ & $53(33.5)$ & $60(38.0)$ & $5.20(3.53-7.64)$ & - & - \\
\hline \multicolumn{8}{|l|}{ Needle injury } \\
\hline No & 1,229 & $23(1.9)$ & $160(13.0)$ & $183(14.9)$ & 1 & 1 & 1 \\
\hline Yes & 445 & $7(1.6)$ & $93(20.9)$ & $100(22.5)$ & $1.66(1.26-2.18)$ & $1.62(1.20-2.18)$ & $1.60(1.19-2.14)$ \\
\hline $\begin{array}{l}\text { Do not } \\
\text { remember }\end{array}$ & 220 & $6(2.73)$ & $30(13.6)$ & $36(16.4)$ & $1.12(0.76-1.65)$ & $1.02(0.66-1.58)$ & $1.14(0.76-1.73)$ \\
\hline \multicolumn{8}{|l|}{ Sex } \\
\hline Females & 103 & $25(1.8)$ & $193(13.8)$ & $193(15.5)$ & 1 & 1 & - \\
\hline Males & 491 & $11(2.2)$ & $90(18.3)$ & $101(20.6)$ & $1.41(1.08-1.83)$ & $1.33(0.95-1.87)$ & \\
\hline \multicolumn{8}{|l|}{ Education } \\
\hline $\begin{array}{l}\text { Low } \\
\text { intermediate }\end{array}$ & 754 & $23(3.0)$ & $142(18.8)$ & $165(21.9)$ & 1 & 1 & - \\
\hline $\begin{array}{l}\text { Higher } \\
\text { intermediate }\end{array}$ & 775 & $12(1.5)$ & 90 (11.6) & $102(13.2)$ & $0.54(0.41-0.71)$ & $0.83(0.60-1.14)^{* *}$ & \\
\hline University & 365 & $1(0.3)$ & $51(14.0)$ & $52(14.2)$ & $0.59(0.42-0.84)$ & - & \\
\hline
\end{tabular}


Continuation of Table 3 HBV markers in Health care workers by professional characteristics

\begin{tabular}{|c|c|c|c|c|c|c|c|}
\hline $\begin{array}{l}\text { Professional } \\
\text { characteristics }\end{array}$ & HCWs* & $\begin{array}{l}\text { HbsAg+ } \\
\text { n (\%) }\end{array}$ & $\begin{array}{l}\text { Anti-Hbs+ } \\
\text { n (\%) }\end{array}$ & $\begin{array}{l}\text { Any } \\
\text { marker + } \\
\mathrm{n}(\%)\end{array}$ & $\begin{array}{l}\text { OR }(95 \% \mathrm{CL}) \\
\text { any marker + }\end{array}$ & $\begin{array}{l}\text { Adjusted OR } \\
(95 \% \text { CL) of any } \\
\text { marker + Full } \\
\text { model }\end{array}$ & $\begin{array}{l}\text { Adjusted OR ( } 95 \% \\
\text { CL) of any marker } \\
+ \text { Simpler model }\end{array}$ \\
\hline \multicolumn{8}{|l|}{ Marital status } \\
\hline Single & 868 & $15(1.7)$ & $95(10.9)$ & $110(12.7)$ & 1 & 1 & \multirow[t]{3}{*}{ - } \\
\hline Married & 966 & $21(2.2)$ & $181(18.7)$ & $202(20.9)$ & $1.82(1.41-2.35)$ & $0.93(0.68-1.26)$ & \\
\hline $\begin{array}{l}\text { Divorced/ } \\
\text { widow }\end{array}$ & 60 & $0(0)$ & $7(11.7)$ & $7(11.7)$ & $0.91(0.40-2.05)$ & $0.41(0.17-0.99)$ & \\
\hline \multicolumn{8}{|l|}{ Year of birth } \\
\hline $1960-74$ & 593 & $5(0.84)$ & $36(6.1)$ & $41(6.9)$ & 1 & 1 & 1 \\
\hline $1950-59$ & 675 & $13(1.93)$ & $95(14.1)$ & $108(16.0)$ & $2.56(1.75-3.76)$ & - & - \\
\hline $1940-49$ & 412 & $11(2.67)$ & $93(22.6)$ & $104(25.2)$ & $4.55(3.04-6.79)$ & $1.45(1.19-1.77)^{* *}$ & $1.42(1.18-1.73)^{*}$ \\
\hline 1916-39 & 214 & $7(3.27)$ & 59 (27.6) & $66(30.8)$ & $6.00(3.82-9.44)$ & - & - \\
\hline \multicolumn{8}{|l|}{ Transfusions } \\
\hline No & 1760 & $33(1.88)$ & $252(14.3)$ & $285(16.2)$ & 1 & 1 & \multirow[t]{3}{*}{ - } \\
\hline Yes & 80 & - & $20(25.0)$ & $20(25.0)$ & $1.73(1.02-2.91)$ & $1.60(0.63-2.13)$ & \\
\hline $\begin{array}{l}\text { Do not } \\
\text { remember }\end{array}$ & 54 & $3(5.56)$ & $11(20.4)$ & $14(25.9)$ & $1.81(0.97-3.38)$ & $1.18(0.46-3.02)$ & \\
\hline \multicolumn{8}{|l|}{ Surgery } \\
\hline No & 1,613 & $30(1.86)$ & $222(13.8)$ & $252(15.6)$ & 1 & 1 & \multirow[t]{3}{*}{-} \\
\hline Yes & 231 & $4(1.73)$ & $51(22.1)$ & $55(23.8)$ & $1.69(1.21-2.35)$ & $1.10(0.74-1.61)$ & \\
\hline $\begin{array}{l}\text { Do not } \\
\text { remember }\end{array}$ & 50 & $2(4.00)$ & $10((20.0)$ & $12(24.0)$ & $1.71(0.88-3.31)$ & $1.27(0.45-3.55)$ & \\
\hline \multicolumn{8}{|l|}{ Teeth care } \\
\hline No & 1,415 & $28(2.0)$ & $216(15.3)$ & $244(17.2)$ & 1 & 1 & \multirow[t]{3}{*}{ - } \\
\hline Yes & 438 & $5(1.1)$ & $58(13.2)$ & $63(14.4)$ & $0.81(0.60-1.09)$ & $0.91(0.66-1.26)$ & \\
\hline $\begin{array}{l}\text { Do not } \\
\text { remember }\end{array}$ & 41 & $3(7.3)$ & $9(21.9)$ & $12(29.3)$ & $1.99(1.00-3.95)$ & $2.53(1.00-6.62)$ & \\
\hline Total & 1894 & $36(1.9)$ & 283 (14.9) & $319(16.8)$ & - & - & \\
\hline
\end{tabular}

*Health care workers; ${ }^{* *}$ OR for every increment of category.

Considering the nurses as the base-line group only student nurses showed a significantly lower OR. No major difference was evident among different services of the hospitals (except for the outpatient clinic, where older personnel no longer assigned to night duty is usually allocated). Noticeably this holds true for surgical and laboratory services as well, which are more frequently exposed to blood. Needle injuries in the last year were a significant risk factor for $\mathrm{HBV}$ infection. Personal characteristics showed that female sex, higher education, birth in recent decades (younger age) are "protective", whereas being married, past transfusions and major surgery were risk factors of HBV infection. It has, however, to be recognized that these factors are connected with employment. Sex, for example, is connected with employment: only $13.7 \%$ of nursing staff are males; conversely, $72.4 \%$ of physicians are males, with congruent education levels: two-thirds of HCWs with a university degree are males.

After adjustment for confounders (Table 3), general hospitals showed a lower and significant OR than in univariate analyses; sig- 
nificant linear increase of OR for each category of longer employment and of birth cohort was confirmed; the effect of employment as physician, outpatient clinic, male sex, high education, being married, past transfusion and surgery was no more evident whereas "other" services were a significant exposure (negative confounding was removed). Fitting of the second, simpler model, eliminating all personal factors except for birth cohort, did not produce any major differences.

\section{Discussion}

The present sero-epidemiological study demonstrates that general hospitals carried a significantly higher risk of $\mathrm{HBV}$ infection among HCWs than paediatric hospitals after adjustment for potential confounders.

Lower prevalences were found in two surveys of general hospitals of middle Italy at the same prevaccination screening of the present study $(33.7 \%$ and $23.3 \%$, respectively); significant risk factors were male sex, age, history of dental treatment, blood transfusions, or needlestick injuries, work as surgeons and as nurses $(14,15)$. HBsAg and any HBV marker prevalence were distinctly higher in south Italy (4.8\% and respectively $42.4 \%)$, without significant difference in comparison of workers of an electric plant (16). Surprisingly, the HBsAg prevalence was found to be lower in Rumanians HCW than in general population $(2.1 \%$ versus $5.6 \%)$ (17), while reached $8.1 \%$ in another country of Balkans (18).

We found a much higher prevalence than in a North-American paediatric hospital (9.6\%) (19). In Toronto 10\% of HCWs with frequent blood exposure showed a positive marker versus $2 \%$ without, and at the final multivariate analysis the strongest determinant was not the hospital employment but the birth place outside North America/United Kingdom (20).

Increasing age, male sex, and non-white ethnicity were significantly, independently associated with higher prevalence of anti$\mathrm{HBs} / a n t \mathrm{i}-\mathrm{HBc}$ in another paediatric hospital, and occupational group, years of work, blood or patient contact, foreign country of birth and education level had no association. Overall prevalence was $12.5 \%$ and the comparison with blood donors, excluding HCWs with history of hepatitis, disclosed an higher prevalence only in older physicians (>40 years-old) (21), and needle injury was confirmed as an independent significant risk factor of HBV infection.

Our results seem to confirm the hypothesis raised by King et al. that a lower risk of $\mathrm{HBV}$ infection in paediatric hospitals is due to different patients cared for (21). Major strengths of the present survey are completeness of the mandatory screening, and same population background risk of $\mathrm{HBV}$ infection. The first one eliminates potential selection biases, due to different compliance of differently exposed HCWs, and the second permits an unbiased comparison.

Data on professional and personal risk factors were collected before the outcome (HBV positive markers) was known; the only possible reason for prior knowledge of HBV markers in absence of past history of hepatitis would be occasional finding of abnormal ALT in HBsAg carriers, but none of HBsAgpositive HCWs showed abnormal ALT. So a recall bias can be confidently excluded. Moreover, the screening was conducted in the context of a vaccination campaign, not addressing any specific hypothesis, and also interviewer information bias can be excluded. Moreover, even if our results are gathered in a specific context, they apply in populations not exposed to anti-HBV vaccination and can suggest risk factors potentially useful in a global perspective.

\section{Limitation of study}

Some limitations of the study need to be noted. No adjustment for birthplace was possi- 
ble, an important risk factor for higher HBV infection in the south of Italy (12). However, there is no reason to assume a difference in geographic origin between HCWs of general and those of paediatric hospitals. Since anti-HBc was not tested, underestimation of HBV infection can be presumed $(20,22)$. A comparison of the present study's HBV prevalence $(12.5 \%)$ can therefore only be made with a similar study in Toronto (7.7\%) (21). However, in the latter study only volunteers were examined ( $25.7 \%$ of HCWs), with possible selection of more conscious HCWs, who can adopt more stringent precautions than non-participants. Storch found exactly the same prevalence as the present survey $(12.5 \%)(22)$ testing anti-HBs and anti-HBc but not HBsAg.

Another potential concern in our study is misclassification of area of work: HCWs can be allocated to a different area of the hospital, so attributing the HBV infection risk to the final department at the time of the survey, and obscuring the effect of an exposure in the original service. This can bias the OR of the exposure toward null if the chance of changing department is the same whatever the $\mathrm{HBV}$ infection status (non-differential misclassification). The only situation that could have resulted in differential misclassification would have been the removal of HBsAg carriers from a high-risk area to a service where exposure of patients is minimized; only seven HCWs with a known history of clinical hepatitis B were potentially infectious ( $\mathrm{HBsAg}$ positive), and only two were in "Other services", where they had been placed after reallocation from clinical services, whereas the other five still worked in clinical services. It is unlikely, therefore, that a differential misclassification played a major role in the results of this study. Lastly, the professional risk of infection was confined to needlestick, so omitting other means of injuries from sharp instruments in the laboratories or surgical theaters, like scissors or lancets.

\section{Conclusion}

In conclusion, the present survey shows that HCWs of paediatric hospitals are less exposed to HBV infection than those of general hospitals. A unique feature of the study is completeness of enrollment in the screening. Its results can be used to target vaccinal policies in countries without universal vaccination against $\mathrm{HBV}$. In developed countries, where vaccines have gained a wide diffusion, susceptible HCWs can be yet unprotected for incomplete coverage, due to poor individual acceptance.

Aknowledgments: Part of the paper has been presented as the dissertation for the Master degree in Epidemiology at London School of Hygiene with the tutoring of Dr Krina Zondervan, and has been revised to update it; Caterina Di Felice has helped to gather the data.

Authors' contributions: Conception and design: VDC; Acquisition, analysis and interpretation of data: GC, VDC; Drafting the article VDC; Revising it critically for important intellectual content: GC, VDC.

Conflict of interest: The authors declare that they have no conflict of interest.

\section{References}

1. Mahoney FJ. Update on diagnosis, management, and prevention of hepatitis B virus infection. Clin Microbiol Rev. 1999;12:351-66.

2. Sherlock S. Hepatitis B: the disease Vaccine 1990;8(Suppl.):S6-10.

3. Alter MJ. Epidemiology of hepatitis B in Europe and worldwide. J Hepatol 2003;39:S64-S69.

4. Pruss-Ustun A, Rapiti E, Hutin Y. Estimation of the global burden of disease attributable to contaminated sharps injuries among health-care workers. Am J Ind Med. 2005;48:482-90.

5. Albertoni F, Ippolito G, Petrosillo N, Sommella L, Di Nardo V, Ricci C, et al. Needle stick injury in hospital personnel: a multicenter survey from central Italy. Infect Control Hosp Epidemiol 1992;13:89-93.

6. Astbury C, Baxter P. Infection risks in hospital staff from blood: hazardous injury rate s and acceptance 
of hepatitis B immunization. J Soc Occup Med. 1990;40:92-3.

7. Jackson MA, Williams K, Burgess CO, Kinney J, Olson LC, Burry VF. Needlestick injuries in a pediatric hospital. Pediatr Infect Dis J. 1994;13:318-9.

8. Pettit L, Gee S, Begue R. Epidemiology of sharp object injuries in a children's hospital. Pediatr Infect Dis J. 1997;16:1019-2.

9. Nambiar S, Singh N. Sharps injuries in a paediatric teaching hospital: a shared responsibility Arch Pediatr Adolesc Med.2001;155:1277-78.

10. Beltrami EM, Williams IT, Shapiro CN, Chamberland ME. Risk and management of blood-borne infections in health care workers. Clin Microbiol Rev. 2000;13:385-407.

11. Ott JJ, Stevens GA, Groeger J, Wiersma ST. Global epidemiology of hepatitis B virus: new estimates of age-specific $\mathrm{HBsAg}$ seroprevalence and endemicity. Vaccine. 2012; 30:2012-219.

12. Pasquini P, Kahn HA, Pileggi D, Panà A, Terzi J, Guzzanti E. Prevalence of hepatitis B markers in Italy. Am J Epidemiol 1983;118:609-709.

13. Stroffolini T, Chiaramonte M, Craxì A, Franco E, Rapicetta M, Trivello R, et al. Baseline seroepidemiology of hepatitis $\mathrm{B}$ virus infection in children and teen-agers in Italy. A survey before mass hepatitis B vaccination. J Infect. 1991;22:191-99.

14. Pasquini P, Kahn HA, Pileggi D, Menichella D, Maddaluno R, Perroni L, et al. Hepatitis B in two italian hospitals. Boll Ist Sieroter Milan 1983;82:308-16.

15. Petrosillo N, Puro G, Ippolito V, Di Nardo V, Albertoni F, Chiaretti B, et al. Hepatitis B virus, hepatitis $\mathrm{C}$ virus and human immunodeficiency virus infection in health care workers: a multiple regression analysis of risk factors. J of Hosp Infect. 1995;30:273-81.
16. Antoniello S, Auletta M, Cerini R, MemoliA, Cogolari S, Quagliata L, et al. Hepatitis B virus infection among health care workers at an urban teaching hospital in southern Italy: a low occupational hazard? Eur J Epidemiol. 1989;5:228-33.

17. Voiculescu M, Iliescu L, Ionescu C, Micu L, Ismail G, Zilisteanu D, et al. A cross-sectional epidemiological study of HBV, HCV, HDV and HEV prevalence in the SubCarpathian and South-Eastern regions of Romania. J Gastrointestin Liver Dis. 2010 Mar;19(1):43-8.

18. Voiculescu M, Iliescu L, Ionescu C, Micu L, Ismail GZilisteanu D, et al. A cross-sectional epidemiological study of HBV, HCV, HDV and HEV prevalence in the subcarpathian and south-eastern regions of Romania. J Gatrointestin Liver Dis. 2010;19:43-8.

19. Kondili LA, Ulquinaku D, Hajdini M, Bashoa M, Chione P, Madonna E, et al. Hepatitis B virus infection in health care workers in Albania: a country still highly endemic for HBV infection. Infection. 2007;35:94-7.

20. Christenson B. Acute infections with hepatitis B virus in medical personnel during a 15 -year followup. Am J Epidemiol 1985;122:411-17.

21. Hicks RA, Cullen JW, Jackson MA, Burry VFC. Work-related risk factors for hepatitis B virus infection in personnel of a children's hospital. Clin Pediatr (Phila). 1989;28:245-50.

22. King SM, Jarvis DA, Shaw J, Shannon HS, Middleton PJ, Gold R, et al. Prevalence of hepatitis $\mathrm{B}$ surface antigen and antibody (hepatitis $\mathrm{B}$ virus markers) in personnel at a children's hospital. Am J Epidemiol. 1987;126:480-83.

23. Storch GA, Perrillo RP, Miller JP, Benz B, Kahn RA. Prevalence of hepatitis B antibodies in personnel at a children's hospital. Pediatrics. 1985;76:29-35. 